\title{
Les théories fondatrices de la finance comportementale
}

\author{
My Hicham SNINEH ${ }^{1}$ et Hicham MESK ${ }^{2}$ \\ ${ }^{1}$ Faculté des Sciences Juridiques, Économiques et Sociales Ain Chock, Université Hassan II, Casablanca, Maroc \\ Laboratoire : Business Intelligence, Gouvernance des Organisations, Finance et Criminalité Financière \\ ${ }^{2}$ Faculté des Sciences Juridiques, Économiques et Sociales Ain Chock, Université Hassan II, Casablanca, Maroc \\ Laboratoire : Business Intelligence, Gouvernance des Organisations, Finance et Criminalité Financière
}

\begin{abstract}
Résumé/Abstract : Les apports de la finance comportementale ne sont actuellement plus à démontrer. La recherche en termes des sciences cognitives a justement permis de mettre en évidence l'existence de biais et heuristiques qui -entre autres- conditionnent les décisions des investisseurs en bourse. Ainsi, celles-ci ne sont pas nécessairement parfaitement rationnelles, contrairement à ce que stipule la finance classique. Diverses et éparpillées dans la littérature y afférente, les théories fondatrices de ces deux courants font dans ce papier l'objet d'un tri, d'un recueil synthétique des principales d'entre elles, puis d'une confrontation franche mettant en évidence leurs points de divergence et leurs aspects complémentaires.
\end{abstract}

Mots-clés : Perspectives; utilité ; rationalité limitée ; comportements suivistes ; inversion des préférences.

\section{Introduction}

La finance comportementale représente une discipline de recherche relativement récente, basée sur la psychologie. Les individus considérés dans les modèles qu'elle propose optent pour des choix d'investissement en présence de risques ou d'incertitudes. L'analyse de leurs comportements y trouve tout son sens.

Dans ce qui suit, nous présentons d'abord les principales théories basées sur les perspectives, développées dans le cadre de la recherche en termes de finance comportementale, puis celles relatives à l'utilité, base fondamentale de la finance classique.

\section{Théories basées sur les perspectives}

Deux grandes théories sont mobilisées à ce niveau : la théorie des perspectives et la théorie des perspectives cumulées. Elles ont été enrichies par des travaux de recherche mettant en évidence l'existence des heuristiques et des biais individuels comportementaux, qu'ils soient émotionnels ou cognitifs (relatifs à l'information).

Si la théorie des perspectives est l'élément fondateur de la finance comportementale, celle des perspectives cumulées en représente le prolongement naturel, développé après plusieurs années de recherche. Les deux théories sont le fruit des travaux des mêmes auteurs : Kahneman et Tversky. 


\subsection{Théorie des perspectives}

Point de départ des recherches devenues aujourd'hui un courant de pensée à part entière, la théorie des perspectives a été développée en 1979 par Kahneman et Tversky, considérés comme les vrais fondateurs de la théorie financière comportementale.

Les travaux de recherche en la matière se sont succédés depuis cette époque, jusqu'à obtenir une certaine reconnaissance au sein de la communauté scientifique. Il en témoigne les Prix Nobel d'économie décernés à plusieurs auteurs, notamment celui qui a été octroyé à Kahneman en 2002 (Tversky, étant décédé avant cette date, n'a pu être conjointement décoré).

La théorie des perspectives puise son fondement dans la neuroscience. Cette discipline observe le cerveau via les techniques proposées par l'imagerie fonctionnelle. L'objectif est de détecter les signaux que le cerveau produit quand il est en pleine action. Avec l'avancée sans cesse croissante de ces techniques, cette démarche exploratoire permet de mieux en mieux repérer quelles zones du cerveau sont excitées et donc mobilisées lorsque l'individu exécute une opération d'ordre cognitif.

Particulièrement, la neuro-économie est une discipline qui en est issue et qui consiste à se servir de l'imagerie cérébrale fonctionnelle pour dépister les origines des prises de décision faites sur les marchés, par des agents amenés à choisir entre plusieurs situations [1].

La principale conclusion faite dans le cadre de la théorie des perspectives stipule que la décision prise par un individu ne peut pas être objectivement rationnelle, comme il était acquis jusqu'aux années 1970. La découverte qui démontre ce postulat confirme que le cerveau chez l'être humain héberge et mobilise de manière consécutive deux mécanismes de pensée, dits respectivement instinctif et rationnel [2]. L'instinct rappelle ici la réactivité, tandis que la rationalité se traduit par la réflexion lente et plus ou moins maîtrisée.

Schématisons cette double configuration de manière simple. Lorsqu'un individu est amené à prendre une décision, c'est le premier mécanisme qui se déclenche d'abord et lui procure une première sensation automatiquement [2] et surtout rapidement [1]; les décisions étant des résultats spontanés suite à l'urgence d'agir [3]. Juste après, le deuxième mécanisme est activé et lui offre le résultat d'une analyse plus approfondie [2].

Dans la réalité, nous pouvons confirmer ce processus car nous sommes chaque jour témoins d'expériences pareilles.

À titre d'illustration, mettons un bébé âgé de quelques mois, et qui connait la notion de hauteur, au bord d'une toiture faite en vitre transparente, et mettons un jouet à l'autre bord. Dans un premier temps, le bébé ne bougera pas. Du fait de la profondeur qu'il va apercevoir à travers la vitre et du risque de chute, il aura peur, comme première sensation instinctive et systématique. Quelques secondes, voire quelques minutes plus tard où il aura analysé sa situation, il décidera de ramper sereinement sur la vitre pour rejoindre le jouet.

Après un moment de concentration et de réflexion un peu plus lent, le bébé aura compris d'une certaine manière que même si la vitre est transparente, elle constitue finalement une substance réellement existante et une base solide sur laquelle il peut traverser sans risque.

Justement, c'est cette fragmentation de la pensée que les comportementalistes considèrent comme origine des troubles qui biaisent éventuellement l'appréciation faite par un individu [1]. 
Plus encore, dans l'exemple précédent, au moins le bébé avait le temps pour réfléchir et la possibilité de toucher la vitre, de la tester et de découvrir ses attributs, avant de décider. Le marché boursier, par contre, met l'investisseur dans des situations risquées beaucoup plus complexes à assimiler. A défaut de pouvoir tout comprendre et analyser dans le temps, la décision qui serait issue du premier système de pensée risque d'être inadaptée, d'où le concept des comportements non parfaitement rationnels.

Notons que notre choix de concevoir cette illustration où l'agent est un bébé n'est pas réducteur. $\mathrm{Au}$ contraire, nous avons intentionnellement voulu mettre la lumière sur les mécanismes universels de l'activité cérébrale humaine à leur état pur. D'autant que l'être humain, quel qu'en soit l'âge, est animé naturellement en partie par son instinct.

Consolidons la présente analyse par un autre exemple fréquent, impliquant cette fois-ci un individu adulte. Imaginons que cette personne soit dans un zoo, devant la cage verrouillée des lions. Si un lion avançait brusquement en direction de l'individu en question, ce dernier aurait tendance à reculer rapidement voire à s'éloigner, par peur. Il savait certes à l'avance qu'il était en situation de sécurité, mais son instinct l'amènera à agir ainsi, comme première réaction. La deuxième réaction, avec du recul réfléchi, le fera revenir près de la cage pour continuer à contempler le spectacle.

Cette posture représente le noyau de la théorie des perspectives dont le modèle est proposé comme une alternative à celui de l'utilité espérée (décrite dans le titre suivant). En proposant une description des choix risqués à travers plusieurs expériences menées, Kahneman et Tversky estiment que -vu ce qui précède- les individus font forcément des erreurs de jugement lors des calculs probabilistes qu'ils sont amenés à faire.

Mais, dans les deux exemples précédents, il est remarqué un même processus : l'apprentissage. Le vécu conditionne les décisions de l'individu mais pas nécessairement ses premières réactions.

Si initialement Kahneman et Tversky n'avaient pas nécessairement l'intention de créer un nouveau paradigme de la finance, il n'en reste pas moins que leur analyse critique vis-à-vis de la rationalité, appuyée par des expérimentations diverses, représente un pilier fondamental pour la recherche en finance. Leur apport a amélioré considérablement la précision et la qualité de la description du jugement humain.

Mais, dans le temps, les critiques dans ce sens ont des origines lointaines.

Déjà, Nicolas Bernoulli a introduit le paradoxe de Saint-Pétersbourg selon lequel le calcul mathématique ne peut à lui seul constituer la logique d'une décision. Ledit paradoxe a été repéré dans la formulation de l'espérance mathématique proposée par Pascal lorsque le Chevalier de Méré lui a demandé une solution rationnelle pour résoudre les jeux de hasard. Équivalente à l'utilité espérée, il s'agit en effet de la moyenne des gains possibles pondérés par les probabilités de leurs occurrences respectives, à travers laquelle on pourrait estimer la somme d'argent initiale à miser. [4]

Pourquoi Nicolas Bernoulli trouve-t-il cela paradoxal ? Tout simplement parce que l'espérance de gain calculée est toujours positive et peut parfois être infinie. Or, l'individu, étant averse au risque, ne peut mettre en jeu une somme d'argent sans limite.

Daniel Bernoulli, lui, enrichit cet aspect en mettant en évidence la différence entre la somme d'argent misée et son utilité qu'il qualifie de "valeur psychologique" [5]. Cette valeur facile à assimiler à travers le concept d'utilité marginale suppose l'existence d'un niveau de référence [4]. 
Élément fondamental de la théorie des perspectives, cette même notion que Kahneman et Tversky qualifient de "perception sensorielle", consiste à ce que le sentiment de déception issu de la perte d'une somme d'argent soit plus intense que celui de satisfaction ressenti lors du gain d'une somme équivalente [6]. Plus précisément, il faudrait gagner deux unités monétaires et demi $(2,5)$ pour compenser une (01) unité monétaire perdue [2]. Cela rend psychologiquement asymétriques les perspectives de gain et celles de perte, contrairement à la distribution Normale que la théorie de l'utilité espérée permet de comprendre.

Enfin, [7] estime que les dysfonctionnements des marchés financiers montrent l'incapacité de la finance à se réinventer, ou à produire de nouveaux instruments viables. Les techniques de placement devraient alors s'adapter aux circonstances extérieures souvent changeantes [8].

Inspiré des travaux menés par Taleb et Mandelbrot, le nouveau paradigme de la finance tel qu'il est vu par [7] a mis en évidence cet aspect d'asymétrie en considérant l'existence non négligeable d'évènements extrêmes. Tenir compte de la non-normalité des rendements améliore l'efficience de l'allocation des actifs financiers [9].

Mandelbrot affirme que considérer les lois de puissance au lieu de la distribution gaussienne améliore la prédictibilité en remédiant aux lacunes de la moyenne qui neutralise les valeurs extrêmes [7]. Son modèle dit multifractal montre que la loi Normale n'est pas la seule à pouvoir décrire les actifs financiers. De ce fait, des éléments subjectifs, souvent d'ordre qualitatif comme les circonstances propres à chaque investisseur, peuvent le guider vers une allocation adéquate [9].

Taleb, lui, propose un portefeuille qu'il estime plus sûr que celui établi par Markowitz, et qui favorise la survenance d'évènements positifs, en comprenant à la fois des titres sans risque et d'autres avec risque élevé, au lieu de contenir des actifs à risque moyen calculé. [7]

\subsection{Théorie des perspectives cumulées}

Développée par [10], la théorie des perspectives cumulées constitue un prolongement de la théorie des perspectives et en représente une variante. La nouveauté réside dans le fait que c'est la fonction de distribution de probabilité cumulée qui est pondérée, au lieu que ce soit les probabilités des résultats pris individuellement.

Dans ce sens, il s'agit d'un effet de cadrage selon lequel le niveau de référence, auquel les investisseurs comparent les résultats probables, est quasiment fixe. Il s'agit d'un point de départ plutôt qu'un point d'arrivée [10].

Par conséquent, vu que l'aversion au risque diffère d'un individu à un autre, les agents peuvent espérer des gains au-delà du niveau de référence ou situer leurs pertes probables en-deçà du point de référence [6]. En outre, les pertes probables sont prises en compte plus que les gains possibles [11].

[10] estiment en plus que les agents tendent à surestimer les pondérations attribuées aux événements extrêmes, même dans le cas où ces derniers sont peu probables.

Enfin, de par sa pertinence et l'amélioration qu'elle a pu proposer, la théorie des perspectives cumulées a été largement utilisée par divers centres de recherche et sur plusieurs situations, économiques notamment, et a été validée en conséquence.

Elle a également validé d'anciennes expérimentations menées par [12] sur les souscripteurs à une assurance dont la probabilité de non remboursement est faible, soit $1 \%$. D'après les réponses des 
personnes questionnées, ceux-ci ne sont disposés à payer que très peu pour s'assurer le complément en vue d'un remboursement complet, et ce, contrairement à ce que stipule la théorie de l'utilité espérée.

En somme, les théories basées sur les perspectives des individus sont venues mettre en évidence les lacunes affichées par les théories basées sur le concept d'utilité.

\section{Théories relatives à l'utilité}

La finance classique a été depuis longtemps analysée dans les divers travaux de recherche à travers une approche normative, dite aussi prescriptive. Selon cette approche, les individus sont pris pour bien informés, aptes à opérer des calculs complexes, précis et rationnels.

Dans ce sens, les chercheurs ont essayé d'expliquer comment ces individus optent pour des choix d'investissement selon des comportements théoriques anticipés.

Deux grandes théories se distinguent ici. Il s'agit d'abord de la théorie de l'utilité espérée où les décisions sont rationnelles face à des opportunités risquées. Il s'agit ensuite de la théorie de l'utilité subjective selon laquelle les décisions sont fondées sur des calculs statistiques et probabilistes complexes.

Dans un contexte de certitude, il est normal de reconnaitre que les agents fassent des choix façonnés par la recherche d'un maximum de satisfaction. Ils utilisent alors une fonction d'utilité, et leurs décisions sont supposées être "parfaites".

Qu'en est-il alors dans un contexte d'incertitude?

En utilisant des calculs probabilistes, les théories relatives à l'utilité apportent à ce questionnement des éléments de réponse différents, selon qu'il s'agisse de risque ou d'incertitude.

\subsection{Théorie de l'utilité espérée}

Quand les probabilités allouées aux variables aléatoires d'une situation sont connues, l'agent fait donc face à une situation risquée. C'est la théorie de l'utilité espérée qui est mobilisée dans ce cas.

La théorie de l'utilité espérée a été développée conjointement par Von Neumann et Morgenstern en 1944. Mais en tant que nouveau concept relevant de la microéconomie, elle a été introduite pour la première fois par Bernoulli en 1937.

Le modèle y afférent permet de décrire le comportement d'un individu en supposant que ses décisions sont rationnelles face à des opportunités risquées, comme dans les jeux de loterie. Von Neumann et Morgenstern développent ainsi l'élément fondateur de la théorie des jeux, d'où l'intitulé de leur ouvrage.[13]

Concrètement, comme son nom l'indique, la théorie de l'utilité espérée stipule que l'agent affecte une valeur à son degré de satisfaction (ou d'utilité) espéré pour chaque situation dans laquelle il se trouve [13]. Le concept de préférence trouve ici tout son sens.

Ainsi, entre deux loteries, il choisira évidemment celle dont l'utilité espérée affiche la valeur supérieure. Par analogie, devant plusieurs portefeuilles, il choisira celui qui lui procurera un maximum de rendement.

En somme, la théorie de l'utilité espérée repose sur les quatre axiomes ci-après.

\subsubsection{Axiome d'invariance}


Selon ce principe, les choix ne varient pas selon la manière dont les options sont présentées ou décrites. Ils en sont indépendants. Autrement dit, si l'on présente à un individu deux versions différentes d'un même jeu à options, les préférences qui en découleront seront identiques.

Pourtant, bien qu'il soit fondamental pour la modélisation des choix rationnels, ce principe est critiqué par plusieurs chercheurs, notamment [14] puis [2].

\subsubsection{Axiome de simplification}

Il s'agit d'un principe intuitif dont la formalisation a été faite par [15] à travers ce qu'il appelle le principe de la chose sûre. Mais avant lui, [16] l'avaient introduit à travers l'axiome de substitution qui amène l'individu à se simplifier les choix en éliminant les éléments communs dans les options proposées.

\subsubsection{Axiome de transitivité}

Cet axiome relatif au modèle de la rationalité consiste en l'existence de préférences transitives chez les individus. Cela veut dire que si une option $\mathrm{A}$ est préférée à l'option $\mathrm{B}$, et si cette dernière est préférée à une autre option $\mathrm{C}$, alors l'option $\mathrm{A}$ est nécessairement préférée à l'option $\mathrm{C}$.

Cependant, [17] a démontré que cette logique de transitivité ne s'applique pas forcément aux décisions d'un individu ou d'un groupe d'individus donnés.

\subsubsection{Axiome de dominance}

Selon cet axiome, si une option $\mathrm{A}$, comparée à une option $\mathrm{B}$, est jugée meilleure sur tous les plans, alors l'option A est dite dominante, d'où le nom de l'axiome. Par conséquent, l'option A est naturellement préférée à l'option $\mathrm{B}$.

Bien qu'il ne soit pas validé par [18], l'axiome de dominance reste solide de par son caractère intuitif.

\subsection{Théorie de l'utilité subjective}

Contrairement à ce que considère la théorie de l'utilité espérée, quand les probabilités allouées aux variables aléatoires d'une situation ne sont pas connues, l'agent est face à l'incertitude. C'est la théorie de l'utilité subjective qui est mobilisée dans ce cas.

Développée par [15], c'est une théorie selon laquelle les décisions sont fondées sur des calculs probabilistes complexes et conditionnées par les règles du jeu en vigueur sur le marché. L'incertitude provient donc de deux sources : d'abord du comportement des autres agents, puis des autres facteurs exogènes.

Par conséquent, non seulement les préférences sont tenues en compte, mais également les attitudes face au risque des agents pris individuellement. Ce ne sont que les choix incohérents et les erreurs de jugement qui s'expliquent par la non rationalité parfaite des individus.

Pour [15], la fonction d'utilité à maximiser permet dans ce cas de tenir en compte l'aversion au risque des agents, dont les croyances sont mesurées par des probabilités subjectives.

Comme nous venons de voir, la finance comportementale regroupe un ensemble important de travaux de recherche menés sur plusieurs décennies, et bâtis essentiellement sur l'aspect psychologique des agents. Plus particulièrement, l'objet étudié relève de la psychologie sociale qui s'intéresse aux diverses formes d'interaction entre agents structurés en groupe. 
[19] propose dans ce sens d'analyser le marché à travers les règles d'interaction des agents. Pour lui, ces règles peuvent changer suite à l'évolution des logiciels et des techniques de communication utilisés.

Les théories qui en ont découlé apportent des enseignements intéressants, constituant une grille d'analyse nouvelle qui permet de mieux comprendre le processus de formation des prix sur le marché.

\section{Théories d'ordre spécifique}

La liste des théories citées ci-après n'est pas exhaustive. Nous avons choisi intentionnellement les théories qui peuvent être apparentes de manière évidente lors de la différenciation entre les divers profils d'agents présents sur un marché.

\subsection{Théorie de la rationalité limitée}

La théorie de la rationalité limitée, comme son nom l'indique, stipule que la rationalité parfaite est impossible à observer dans la réalité. Le travail de recherche qui a abouti à cette conclusion a valu à son auteur, Herbert Simon, le prix Nobel d'économie en 1978.

[20] a décomposé le processus de prise de décision en trois grandes étapes. D'abord l'observation de la problématique, ensuite le choix des options à prendre et enfin l'appréciation des résultats de la mise en œuvre des options choisies.

En analysant empiriquement les conditions de prise de décision, cette décomposition a permis de détecter un certain nombre de facteurs qui empêchent de prendre une décision objective. Il s'agit -entre autres- du temps réduit, de la non disponibilité de toutes les informations et de certains biais cognitifs.

Le principal parmi ces biais est l'inaptitude de l'individu à assimiler tout ce que son environnement lui présente, l'amenant ainsi à chercher des options simples plutôt que des solutions optimales [20]. Par conséquent, son degré de satisfaction serait juste acceptable au lieu d'être maximal, ce qui ne vérifie pas la rationalité parfaite telle qu'elle est définie dans les modèles moyenne-variance.

La rationalité n'est donc plus vue comme économiquement parfaite. Elle cède la place à une rationalité psychologique limitée et donc plus réaliste.[21]

\subsection{Théories des comportements suivistes}

Sans vouloir insinuer une connotation péjorative, quel que soit le caractère des individus, il y en aura toujours une fraction d'agents qui tendent à se suivre. La logique du troupeau existe effectivement sur le marché, notamment quand les spéculations se forment [22].

En tous cas, le suivisme est une attitude qui a plusieurs formes semblables à nuancer.

\subsubsection{Contagion des idées}

Le fait d'investir ou de s'abstenir, de choisir telle option et non pas une autre dépend évidemment de plusieurs considérations.

Une parmi ces considérations vient des idées des autres. Dans ce sens, une certaine contagion d'idées se répand entre les agents lorsqu'ils s'engagent dans des dialogues, ce qui a une influence sur leurs décisions d'investissement respectives [22]. 
La contagion la plus observée sur le marché reste celle relative aux pratiques spéculatives [23] car la formation des bulles y afférentes représente l'illustration parfaite de ce phénomène.

Ladite contagion est d'autant plus intense et rapide de nos jours avec l'usage de plus en plus croissant des moyens technologiques de communication. C'est le cas des forums et des diverses plateformes de discussion que l'internet propose.

En conséquence, et vu le nombre immense d'informations que l'agent y intercepte, la difficulté pour lui de faire la distinction entre une information et un bruit de fond peut entraîner une variation des prix à la hausse, comparés aux résultats issus des modèles de la théorie financière classique.

Enfin, quand la contagion d'idées est de type à rendre irréversible le processus de formation de l'opinion, nous pouvons parler d'une contagion mimétique [24]. Ce type de contagion rappelle celui des comportements mimétiques.

\subsubsection{Comportement mimétique}

Il s'agit ici d'un phénomène social équivalent à la tendance des agents à s'imiter.

Le marché financier, comme tout marché d'ailleurs, étant un réceptacle d'une multitude d'agents en continuelle interaction, héberge des actions collectives. Ce sont ces actions entreprises collectivement qui reflètent la tendance des agents à se copier les uns des autres.

Certaines recherches sont allées plus loin dans l'analyse jusqu'à démontrer que les gènes qui se transmettent de génération en génération d'investisseurs peuvent transporter l'attitude d'imiter, selon la théorie des mèmes [25], [26] et [27].

\subsubsection{Conformisme}

Plusieurs chercheurs ont confirmé que le conformisme est un phénomène sociétal très observé dans la vie humaine contemporaine. Les besoins qui naissent de la comparaison aux autres amènent l'agent à faire des choix et à prendre des décisions. [28] a déjà confirmé ce constat en mettant en évidence le poids du conformisme sur le processus de réflexion et d'arbitrage chez l'agent au sein de la communauté.

Bien que les motivations des uns ne soient pas les mêmes que celles des autres, les investisseurs tendent curieusement à se conformer aux attitudes du groupe et cela peut être l'origine de plusieurs autres biais[8].

En allant plus loin dans l'analyse, [29] ont utilisé la technique de l'imagerie cérébrale fonctionnelle pour démontrer que le sentiment d'un membre de la communauté d'en être socialement exclu active les mêmes parties du cerveau que celles excitées par la douleur ressentie physiquement.

\subsection{Inversion des préférences ou l'illusion de préférer}

Contrairement aux croyances qui sont généralement fondées par un raisonnement, les préférences sont moins enracinées chez l'individu. Certaines préférences sont même formées par une impression personnelle ou une idée reçue sans pour autant faire l'objet d'une expérience vécue dans le passé.

C'est pourquoi la réalité quotidienne montre facilement qu'il suffit de la survenance d'un fait mineur l'expérimentation- pour voir les préférences changer. D'où le phénomène de l'inversion des préférences. 
Le changement de préférences, selon [30], est fréquent voire systématique pour qu'il soit ignoré ou pris pour déviation aléatoire.

En effet, [18] ont observé que le changement même insignifiant de la manière dont les choix sont présentés, influe considérablement sur les préférences chez les individus, que ces derniers peuvent complètement inverser. C'est ce que l'on appelle effet de contexte ou de formulation.

Sur le marché financier, où l'enjeu économique (gain en termes monétaire) importe autant, les considérations psychologiques personnelles de l'individu font que le contexte particulier des choix proposés illustre parfaitement ses préférences inversées. L'impact de l'effet de contexte sur les préférences est comparé ici à l'impact des perspectives sur les perceptions individuelles [31].

[32] confirme également que l'inversion des préférences est un phénomène qui existe réellement et peut être expliqué par plusieurs facteurs, dont principalement la non transitivité des préférences. Par ailleurs, [33] parlent de préférences imprécises, tandis que [34], ainsi que [35] parlent de préférences aléatoires comme facteurs explicatifs de ce phénomène.

[36], lui, trouve paradoxal le fait que les individus changent complètement leurs décisions, d'où le concept de "Paradoxe d'Ellsberg" qu'il a introduit. Pour l'illustrer, reprenons un résumé de son expérimentation.

Ellsberg a proposé à une cohorte de participants un jeu en deux temps, où il leur a demandé de :

\section{a. choisir entre deux options :}

- L'option A, plutôt certaine, car la probabilité de gagner est élevée. Mais, la somme à gagner est petite.

- L'option $\mathrm{B}$, jugée risquée, car la probabilité de gagner est faible, même si le somme à gagner est grande.

- $\quad$ Résultat : les répondants choisissent majoritairement l'option A, étant la plus sûre.

\section{b. donner une valeur aux deux options :}

- Résultat : les répondants choisissent majoritairement l'option B, procurant le plus grand gain, en lui attribuant une valeur supérieure à celle attribuée à l'option $\mathrm{A}$.

Paradoxalement, la majorité des participants au jeu proposé par Ellsberg inversent leurs choix initiaux. Justement, pour [37] qui ont repris cette même expérience, les participants ont opté pour l'option la plus sûre lorsqu'ils étaient amenés à choisir, puis ont attribué une valeur supérieure à l'option la plus risquée quand il s'agissait d'évaluer, ce qui constitue une violation de la théorie de l'utilité espérée.

Enfin, les théories disponibles sont très nombreuses évidemment. Mais, la logique que le présent enchainement exige nous amène à nous arrêter à ce niveau où l'idée centrale que nous voulons transmettre est complète et suffisamment claire.

\section{Conclusion}

Il n'est plus à démontrer aujourd'hui que le comportement rationnel n'est ni une attitude toujours observée ni une caractéristique intrinsèque chez tous les agents constituant le marché. Il en est de même pour l'existence de biais comportementaux de plusieurs ordres affectant les décisions des investisseurs dans de larges proportions. [38] 
Et si la communauté scientifique a fini par reconnaitre l'apport de la finance comportementale, c'est parce que celle-ci est fondée sur plusieurs expérimentations solides, puisant dans les résultats des recherches en sciences cognitives, rendant les modèles qui en sont issus plus proches de la réalité. [38]

Il en découle des théories nouvelles, orientées vers les perspectives relatives à l'investissement, plutôt que vers l'utilité que ce dernier peut procurer.

\section{BIBLIOGRAPHIE}

[1] CAMERER C., LOEWENSTEIN G. and PRELEC D. [2005], "Neuroeconomics: How Neuroscience Can Inform Economics", Journal of Economic Literature, 43 (1): 9-64.

[2] KAHNEMAN D. and TVERSKY A. [1979], "Prospect theory: an analysis of decision under risk", Econometrica, Volume 47, Number 2.

[3] KEYNES J. M. [1936], "The General Theory of Employment, Interest, and Money", Hacourt, Brace \& World, New York.

DERRIENNIC Y. [2003], "Pascal et les problèmes du chevalier de Méré. De l'origine du calcul des probabilités aux

[4] mathématiques financières d'aujourd'hui", Université de Bretagne Occidentale, Laboratoire de Mathématiques, Unité CNRS FRE, 2218.

[5] STEARNS S. [2003], "Daniel Bernoulli (1738) : Evolution and economics under risk", Journal of biosciences, 25. 221-8.

[6] BENARTZI S. and THALER R.H. [1995], "Myopic Loss Aversion and the Equity Premium Puzzle", The Quarterly Journal of Economics, 110(1), 73-92.

[7] HERLIN P. [2010], "Finance - Le nouveau paradigme", Eyrolles, Paris.

[8] RICHARDS T. [2014], "Investing Psychology, The Effects of Behavioral Finance on Investment Choice and Bias", Wiley Editions, USA.

[9] SHEIKH A.Z. and QIAO H. [2009], "Non-Normalité des Rendements", J.P. Morgan Asset Management, Paris.

[10] TVERSKY A. and KAHNEMAN D. [1992], "Progrès dans la théorie de perspective: représentation cumulative de l'incertitude". Journal of Risk and Uncertainty. 5 (4): 297-323.

[11] ABDELlAOUI M. [2000], "Parameter-free elicitation of utility and probability weighting functions", MANAGEMENT SCIENCE/Vol. 46, No. 11.

[12] WAKKER P.P., THALER R.H. and TVERSKY A. [1997],"Probabilistic Insurance", Journal of Risk and Uncertainty, 15(1), 7-28.

[13] VON NEUMANN J. and MORGENSTERN O. [1944], "Theory of Games and Economic Behavior", Princeton University Press.

[14] LICHTENSTEIN S. and SLOVIC P. [1971], "Reversals of preference between bids and choices in gambling decisions", Journal of Experimental Psychology, 89(1), 46-55.

[15] SAVAGE L.J. [1954], "The Foundations of Statistics", New York, John Wiley.

[16] VON NEUMANN J. and MORGENSTERN O. [1947], "Theory of Games and Economic Behavior", (2nd rev. ed.) Princeton University Press.

[17] TVERSKY A. [1969], "Intransitivity of preferences", Psychological Review 76 (1) : 31-48. 
[18] TVERSKY A. and KAHNEMAN D. [1981], "The framing of decisions and the psychology of choice", Science, 211(4481), 453-458.

[19] SORNETTE D. [2003], "Why Stock Markets Crash: Critical Events in Complex Financial Systems", Princeton U. Press, Princeton, N.J., (421 pp.).

[20] SIMON H.A. [1972], "Theories of bounded rationality" in Decisions and Organizations, C.B McGuire and R. Radner (Eds), North Holland, Amsterdam, p.161-176.

[21] SCHINCKUS C. [2009], "La finance comportementale ou le développement d'un nouveau paradigme", Revue d'histoire des sciences humaines, $n^{\circ} 20$, p. 101-127.

[22] SHILLER R.J. [2000], "Irrational Exuberance", The American Journal of Economics and Sociology, 59(3), 537540 .

HUBERMAN G. and REGEV T. [2001], "Contagious Speculation and a Cure for Cancer: A Nonevent That Made Stock Prices Soar", The Journal of Finance, 56(1), 387-396.

[24]

ORLEAN A. [1995], "Bayesian interactions and collective dynamics of opinion : Herd behavior and mimetic contagion", Journal of Economic Behavior \& Organization, Volume 28, Issue 2, Pages 257-274.

[25] DAWKINS R. [1990], "Le gène égoïste", Armand Colin, Dunod, 368 pages.

[26] BLACKMORE S. [2000a], "The Meme Machine", Artificial Societies and Social Simulation, Volume 2.

[27] BLACKMORE S. [2000b], "The Power of Memes", Scientific American. 283. 64-6, 68.

ASCH S.E. [1956], "Studies of independence and conformity: I. A minority of one against a unanimous majority", Psychological Monographs: General and Applied, 70(9), 1-70.

[29] EISENBERGER N. and LIEBERMAN M. [2004], "Why Rejection Hurts: A Common Neural Alarm System for Physical and Social Pain. Trends in cognitive sciences. 8. 294-300.

[30] TVERSKY A. and KAHNEMAN D. [1986], "Rational Choice and the Framing of Decisions", The Journal of Business, 59(4), S251-S278.

[31] THALER R.H. and SHEFRIN H.M. [1981], "An Economic Theory of Self-Control", Journal of Political Economy, $89(2), 392-406$.

[32] SEIDL C. [2002], "Preference Reversal", Journal of Economic Surveys. 16. 621 - 655.

[33] BUTLER D.J. and LOOMES G.C. [2007], "Imprecision as an Account of the Preference Reversal Phenomenon", The American Economic Review, 97(1), 277-297.

[34] SCHMIDT U. and HEY J.D. [2004], "Are Preference Reversals Errors? An Experimental Investigation", Journal of Risk and Uncertainty, 29(3), 207-218.

[35] BLAVATSKYY P. [2009], "Preference Reversals and Probabilistic Choice", Journal of Risk and Uncertainty, Vol. 39 (3), pp. 237-250.

[36] ELLSBERG D. [1961], "Risk, Ambiguity, and the Savage Axioms", The Quarterly Journal of Economics, 75(4), 643-669.

[37] GRETHER D.M. and PLOTT C.R. [1979], "Economic Theory of Choice and the Preference Reversal Phenomenon", The American Economic Review, vol. 69, no. 4, p. 623.

[38] SNINEH M.H et MESK H. [2021], "La finance comportementale: Revue de littérature", International Journal of Financial Accountability, Economics, Management, and Auditing. À paraître. 